\title{
Integrative transcriptomics and metabolomics analyses provide hepatotoxicity mechanisms of asarum
}

\author{
SA CAO $^{1}$, LINTAO HAN ${ }^{2}$, YAMIN LI ${ }^{3}$, SHIQI YAO ${ }^{1}$, SHUAIHONG HOU ${ }^{1}$, SHI-SHI MA ${ }^{1}$, \\ WANGQIANG DAI $^{1}$, JINGJING LI ${ }^{1}$, ZHENXIANG ZHOU ${ }^{1}$, QIONG WANG ${ }^{1}$ and FANG HUANG ${ }^{1}$ \\ ${ }^{1}$ Department of Basic Medicine, Hubei University of Chinese Medicine; ${ }^{2}$ Department of Chinese Medicine Resource and \\ Compound Prescription, Ministry of Education, Wuhan, Hubei $430065 ;{ }^{3}$ Department of Pharmacy, \\ Henan University of Chinese Medicine, Zhengzhou, Henan 450046, P.R. China
}

Received August 15, 2019; Accepted March 18, 2020

DOI: $10.3892 /$ etm.2020.8811

\begin{abstract}
Asarum is frequently applied in combination with other agents for prescriptions in practices of Traditional Chinese Medicine. A number of studies have previously indicated that asarum treatment induces lung toxicity by triggering inflammation. However, the potential effects of asarum in the liver and the underlying mechanisms have remained largely elusive. Therefore, transcriptomics and metabolomics approaches were used in the present study to examine the mechanisms of the hepatotoxicity of asarum. Specifically, mRNA and metabolites were obtained from rat liver samples following intragastric administration of asarum powder. RNA sequencing analysis was subsequently performed to screen for differentially expressed genes (DEGs), and a total of 434 DEGs were identified in liver tissue samples, 214 of which were upregulated and 220 were downregulated. Pathway enrichment analysis found that these genes were particularly enriched in processes including the regulation of p53 signaling, metabolic pathways and bile secretion. To investigate potential changes to the metabolic profile as a result of asarum treatment, a metabolomics analysis was performed, which detected 14 significantly altered metabolites in rat liver samples by gas chromatography-mass spectrometry. These metabolites were predominantly members of the taurine, hypotaurine and amino acid metabolic pathways. Metscape network analyses were subsequently performed to integrate the transcriptomics
\end{abstract}

Correspondence to: Professor Qiong Wang or Professor Fang Huang, Department of Basic Medicine, Hubei University of Chinese Medicine, 1 Huangjiahu West Road, Wuhan, Hubei 430065, P.R. China

E-mail:wq_ccp@hbtcm.edu.cn

E-mail: hf_ccp@hbtcm.edu.cn

Abbreviations: DEG, differentially expressed gene; TCM, Traditional Chinese Medicine

Key words: asarum, hepatotoxicity, transcriptomics, metabolomics, integration and metabolomics data. Integrative analyis revealed that the DEGs and metabolites were primarily associated with bile acid biosynthesis, amino acid metabolism and the p53 signaling pathway. Taken together, these results provide novel insight into the mechanism of asarum-mediated hepatotoxicity, which may potentially aid the clinical diagnosis and future therapeutic intervention of asarum poisoning.

\section{Introduction}

Asarum is derived from the dried root and rhizome of Asarum heterotropoides Fr. Schmidt var. mandshuricum (Maxim) Kitag., Asarum sieboldii Miq. var. seoulense Nakai. or Asarum sieboldii Miq. It is commonly applied in Traditional Chinese Medicine (TCM) due to its anesthetic and analgesic properties (1-4). In addition, it is applied in combination with other TCMs for the treatment of various ailments. Ginger and Asarum application by acupoint sticking therapy have been suggested to improve the clinical symptoms of bronchial asthma (5). However, the usage of asarum is generally not recommended due to its toxicity. Previous studies have detected a number of potentially toxic components in asarum, including safrole, methyl eugenol, aristolochic acids, asarone, 3,5-dimethoxytoluene and benzene derivatives $(6,7)$. These components are associated with toxicity in multiple organs, including the central nervous system (8), kidneys (9) and liver (10). In addition, Asarum is carcinogenic (11). In particular, aristolochic acids in asarum have been previously demonstrated to exhibit significant nephrotoxicity, in addition to mutagenic and carcinogenic effects (12). Safrole has been revealed to be associated with the pathogenesis of hepatocellular carcinoma and to lead to respiratory paralysis $(13,14)$.

A number of previous studies have investigated the mechanism of toxicity mediated by single components contained within naturally occurring medicinal compounds. Yang et al (14) indicated that safrole may inhibit cytochrome 450 enzymes and result in the production of reactive metabolites, in turn leading to the inhibition of enzyme activity and increasing the risk of hepatocellular carcinoma progression. In addition, Patel et al (15) demonstrated that cytotoxicity exerted by $\beta$-asarone was associated with lipid peroxidation and glutathione depletion in hepatocytes. However, since TCMs exhibit 
multi-component, multi-target and multi-pathway characteristics, further research is required to unravel the mechanism of toxicity exerted by a single component within TCM herbal formulations (16). In a previous study, genomics and transcriptomics analyses were performed to reveal the mechanisms of asarum-induced lung toxicity, which were potentially mediated through the adenosine monophosphate-activated protein kinase/NF- $\kappa \mathrm{B}$ and $\mathrm{Bcl} 2$ pathways, in addition to proteins associated with inflammation, leading to an inflammatory reaction (11). However, the safety and efficacy of asarum for clinical use remain poorly understood, since the effect of asarum on the liver remains to be fully elucidated.

The liver is the largest organ that participates in drug metabolism, where it serves an important role in energy, lipid and amino acid metabolism (17). Following liver injury, gene expression and metabolite profiles become aberrantly altered. Transcriptomics analyses study the transcription of all genes in cells at a global level $(18,19)$, whereas metabolomics analyses examine the levels of metabolites in bodily fluids or tissues. Combination of these two analyses results in a large amount of data being generated regarding the overall changes in the metabolic spectrum, as a consequence of alterations in the transcriptome $(20,21)$. In the present study, to assess the underlying mechanism of the hepatotoxicity of asarum, transcriptomics and metabolomics datasets were generated and subsequently integrated to investigate prospective changes in the transcriptional and metabolic profiles of rats treated with asarum. Taken together, the present study aimed to provide novel insight into the mechanism of asarum-mediated toxicity, which may provide approaches to improve the clinical diagnosis and development of therapeutic interventions against asarum poisoning.

\section{Materials and methods}

Chemicals and reagent kits. Alanine transaminase (ALT; cat. no. BQ004A-CR), aspartate transferase (AST; cat. no. BQ006A-CR) and total bilirubin (TBil; cat. no. BQ012A-CR) assay reagent kits were obtained from Rayto Life and Analytical Sciences Co.,Ltd. TRIpure RNA extraction reagentwas obtained from BioTeke Corp. The Agilent Fiehn GC/MS Metabolomics Standards Kit was purchased from Agilent Technologies, Inc. $\mathrm{N}$-methyl-N-(trimethylsilyl) trifluoroacetamide (MSTFA), chlorotrimethylsilane (TMCS) and methoxyamine hydrochloride were of gas chromatography (GC) derivatization grade and were purchased from Sigma-Aldrich; Merck KGaA. Other reagents used in the present study were of analytical grade.

Quality control of asarum. Asarum (cat. no. 201809014) was obtained from Wuhan Hankou National Pharmaceutical Co. Ltd. The volatile components of asarum were analyzed using headspace-solid phase microextraction (HS-SPME) combined with GC-mass spectrometry (GC-MS). In brief, $0.3 \mathrm{~g}$ asarum powder was placed in a 20-ml HS vial to extract volatile components. The sample was heated for $15 \mathrm{~min}$ in a thermostatic bath at $90^{\circ} \mathrm{C}$ for equilibration. The fiber was exposed to the HS for 15 min during the extraction time. Following sampling, the SPME fiber was immediately inserted into the $\mathrm{GC}$ injector and thermally desorbed for $3 \mathrm{~min}$ at $260^{\circ} \mathrm{C}$ in 'split-less' mode. The relative percentage of each component was calculated using the area normalization method where the area of a single peak is divided by the total peak area.

Animals. Previous experiments have demonstrated that $1.35 \mathrm{~g}$ asarum $/ \mathrm{kg} /$ day induces lung toxicity in rats (11). Therefore, possible asarum-induced hepatotoxicity was evaluated at the same dose. Asarum root was first crushed using a pulverizer, following which double-distilled $\mathrm{H}_{2} \mathrm{O}$ was added to prepare a $13.5 \%$ suspension for intragastric administration.

In total, 40 male Sprague Dawley (SD) rats (weight, 200-230 g; age, 40-42 days) were purchased from the experimental animal research center of Hubei Province (license number, ZCXK, Hubei 2015-0018). Following one week of acclimatization at $18-25^{\circ} \mathrm{C}$ and a relative humidity of $60-70 \%$, with natural circadian rhythm and light. The rats had free access to food and water. The rats were divided into the following two groups $(n=20)$ : i) Control; and ii) asarum treatment. Subsequently, asarum (1.35 g/ kg/day) was administered by oral gavage over a 28-day period, whilst the control group was administered equivalent volumes of saline. General parameters of each group of rats, including skin, hair and behavioral activities, were monitored daily throughout the duration of the experiment. Rats were used for $\mathrm{H} \& \mathrm{E}$ staining (3), metabolite testing (14) and for gene expression analysis (3). All experimental procedures were performed in accordance with the guidelines established by the animal ethics committee of Hubei University of TCM (Wuhan, China; approval no. HUCMS201903001).

Measurement of organ coefficient and liver function. Following 28 days of intragastric administration, rats were first anesthetized with $10 \%$ chloral hydrate $(300 \mathrm{mg} / \mathrm{kg})$ by intraperitoneal injection. None of the animals exhibited any signs of peritonitis after the administration of $10 \%$ chloral hydrate. Subsequently, $1 \mathrm{ml}$ blood was obtained from the abdominal aorta and the rats were sacrificed by cervical dislocation. After the confirmation of cardiac and respiratory arrest, in addition to the disappearance of nerve reflex, the liver was obtained from each rat. The liver tissues were then rinsed with normal saline, blotted with filter paper and weighed. The organ coefficient was calculated as the weight of the liver relative to $100 \mathrm{~g}$ of the rat's body weight. To obtain serum samples, whole blood was centrifuged at $1,006.2 \mathrm{x}$ for $15 \mathrm{~min}$ at $4^{\circ} \mathrm{C}$, following which the activities of ALT, AST and TBil were determined using a biochemical analyzer (Rayto Life and Analytical Sciences Co., Ltd.) according to the manufacturer's protocol.

$H \& E$ staining. After the rats were sacrificed, liver samples $(\sim 1.5 \times 1.5 \times 0.3 \mathrm{~cm})$ were stored in $4 \%$ paraformaldehyde solution at $4^{\circ} \mathrm{C}$ for $24 \mathrm{~h}$, embedded in paraffin, sectioned at $4.5 \mu \mathrm{m}$, stained with H\&E as previously described (22) and observed for histopathological changes using standard light microscopy. Histopathological scoring analysis was performed by a pathologist according to protocols previously described (22). Assessment was performed by calculating the sum of the following individual score grades: i) 0 (no findings); ii) 1 (mild); iii) 2 (moderate); and iv) 3 (severe) for each of the following six parameters: i) Cytoplasmic color fading; ii) vacuolization; iii) nuclear condensation; iv) nuclear fragmentation; v) nuclear fading; and vi) erythrocyte stasis. 
Gene expression analysis. In total, 3 liver tissues were selected randomly from each group for high-throughput sequencing. In brief, $0.1 \mathrm{~g}$ liver tissue samples were homogenized using $1 \mathrm{ml}$ TRIzol ${ }^{\circledR}$ according to the manufacturer's protocol to extract total RNA. The RNA was then checked for purity and stability by gel electrophoresis and the concentration was determined using the Agilent 2100 Bioanalyzer (Agilent Technologies, Inc.). The mRNA with polyA structures in total RNA was then enriched using Oligo (dT) magnetic beads (Biomag Biotechnology Co., Ltd.; cat. no. BMDT1000; $4^{\circ} \mathrm{C}$ and 2 min) and fragmented into 200-300 bp pieces by ion disruption using theVAHTS Universal Plus DNA Library Prep Kit For Illumina (Biomag Biotechnology Co., Ltd., ND617) following the manufacturer's protocol. Using RNA as a template, the first-strand complementary (c)DNA was synthesized using random hexamer primers and reverse transcription, and second-strand cDNA was synthesized using the first-strand cDNA as a template with the High-Capacity cDNA Reverse transcription kit (cat. no. 4368813; Thermo Fisher Scientific, Inc.), following the manufacturer's protocol. The synthesized cDNA was purified using Agencourt Ampurebeads (Beckman Coulter, Inc.). The purified cDNA was then prepared using a TruSeq DNA single indexes Set A kit (Illumina Inc.; cat. no. FC-121-2001) and amplified using TruSeq PE Cluster Kit (Illumina, Inc.; cat. no. PE-300-2001) to obtain a cDNA library. The total library concentrations were tested using the Agilent 2100 Bioanalyzer prior to being subjected to paired-end sequencing based on the Illumina HiSeq sequencing platform using next-generation sequencing. The raw sequencing data were first filtered by cutadapt (https://cutadapt.readthedocs. io/en/stable/), following which the filtered high-quality sequence was aligned to the reference genome of the rat. Based on the alignment results, the expression level of each gene was calculated, and the samples were analyzed further in terms of difference, enrichment and cluster analyses. Differentially expressed genes (DEGs) were screened out using the unpaired t-test with $\mathrm{P}<0.05$ as the threshold. The Gene Ontology (GO) platform (http://www.geneontology.org/) and panther version 14 were used to perform functional enrichment analysis of the DEGs (23). The Database for Annotation, Visualization, and Integrated Discovery (DAVID; version 6.8; https://david. ncifcrf.gov/) coupled with the Kyoto Encyclopedia of Genes and Genomes (KEGG; http://www.genome.jp/kegg/pathway. html) was used to determine significant pathways associated with the DEGs (24). Pathways with P-value thresholds of $<0.05$ were considered potential target pathways.

Metabolomics data collection and analysis. After thawing the liver tissue samples at room temperature, $1 \mathrm{ml}$ physiological saline was added to $0.2 \mathrm{~g}$ tissue to disrupt the cells, following which $250 \mu 1$ acetonitrile was added to $100 \mu 1$ liver homogenate for protein precipitation. This sample was then homogenized by ultrasound $(40 \mathrm{kHz})$ in an ice bath for $15 \mathrm{~min}$ and centrifuged at $11,180 \mathrm{x} \mathrm{g}$ for $10 \mathrm{~min}$ at $4^{\circ} \mathrm{C}$ to obtain a $200-\mu 1$ supernatant. The supernatant was transferred to a sample vial and concentrated using a centrifugal concentrator $\left(1,006.2 \mathrm{x}\right.$ g) at $35^{\circ} \mathrm{C}$ for $2 \mathrm{~h}$ to evaporate the solvent. Oximation was performed at $30^{\circ} \mathrm{C}$ for $90 \mathrm{~min}$ following the addition of $40 \mu \mathrm{l}$ methoxyamine pyridine solution $(40 \mathrm{mg} / \mathrm{ml})$. Trimethylsilylation was subsequently performed at $37^{\circ} \mathrm{C}$ for $60 \mathrm{~min}$ following the addition of
$80 \mu \mathrm{l}$ derivatization reagent (MSTFA/TMCS ratio, 100:1 v/v). Subsequently, $10 \mu 1 \mathrm{~d} 27$-myristic acid $(0.75 \mathrm{mg} / \mathrm{ml}$ in n-hexane) was added as a reference compound. The derivatized sample was finally transferred to a $250-\mu 1$ vial for GC-MS analysis.

A 7890B GC system (Agilent Technologies, Inc.), equipped with a 5977B series mass selective detector (Agilent Technologies, Inc.) and a DB-5ms capillary column (30 m x $0.25 \mathrm{~mm}$ x $0.25 \mu \mathrm{m}$; Agilent Technologies, Inc.) was used to analyze the derivatized liver samples. To achieve good separation, the oven temperature was programmed from 60 to $250^{\circ} \mathrm{C}$ at $10^{\circ} \mathrm{C} / \mathrm{min}$ and subsequently held at 60 and $250^{\circ} \mathrm{C}$ for 1 and $10 \mathrm{~min}$, respectively. The injector, source and quadrupole temperatures were set to 250,230 and $150^{\circ} \mathrm{C}$, respectively, whilst the detector voltage was $70.0 \mathrm{eV}$ and the MS spectra were obtained in the mass to charge ratio $(\mathrm{m} / \mathrm{z})$ range of $\mathrm{m} / \mathrm{z}=50.0-600.0$. A positive ionization mode was used. The flow rate of the carrier gas, helium, was $1.1 \mathrm{ml} / \mathrm{min}$ and the solvent delay time was $5.9 \mathrm{~min}$. The injection volume was $1 \mu \mathrm{l}$ and the split ratio was 10:1. The helium was injected at room temperature and 80 psi.

GC-MS raw data acquisitions were performed using the Agilent Masshunter software (Qualitative Navigator B.08.00; Agilent Technologies, Inc.). Spectra deconvolution was performed using the Automated Mass Spectral Deconvolution and Identification System (AMDIS) tool (http://www.amdis. net/) from the National Institute of Standards and Technology (NIST) library. All spectra were normalized to total peak intensity. Metabolites with P-values $<0.05$ and fold changes $>2.0$ were considered biomarkers. All biomarkers were tentatively identified using the retention index, the NIST database (http://webbook.nist.gov/chemistry/) and the Agilent Fiehn GC/MS Metabolomics RTL Library $(25,26)$. Principal component analysis (PCA), partial least squares-discriminant analysis (PLS-DA), relative intensity analysis and pathway analysis were performed using the MetaboAnalyst 4.0 online tool (http://www. metaboanalyst.ca/) (27). R2 represents the fit of the model, and $\mathrm{Q} 2$ represents the prediction rate of the model. The closer the values of R2 and Q2 are to 1, the better the model constructed.

Integrated analysis. Cytoscape 3.6.1 (https://js.cytoscape. $\mathrm{org} /$ ) is a software tool for the visual exploration of biomedical networks composed of metabolic, gene and other types of interactions (28). Specifically, Metscape 3 (http://MetScape. ncibi.org) is a Cytoscape plug-in that allows users to build and analyze networks of genes and compounds, identify enriched pathways from expression profiling data and visualize changes in metabolite data (29). The data obtained for differentially abundant metabolites and DEGs from rats in the control and asarum-treated groups were imported into Metscape to obtain a global understanding of gene and metabolic changes to assess the underlying mechanisms of hepatotoxicity.

Statistical analysis. Measurement data, including the data of organ coefficient, liver function, the histopathology score, DEG analysis and metabolite analysis, were presented as the mean \pm standard deviation. The unpaired t-test was performed for comparison of means between two groups using SPSS 21.0 software (IBM Corp.). In addition, a hypergeometric test was performed for pathway and GO analysis. $\mathrm{P}<0.05$ was considered to indicate statistical significance. 
Table I. Volatile components identified in asarum by headspace-solid phase microextraction-gas chromatography-mass spectrometry.

\begin{tabular}{|c|c|c|c|c|c|}
\hline $\begin{array}{l}\text { Peak } \\
\text { number }\end{array}$ & $\begin{array}{l}\text { Retention } \\
\text { time (min) }\end{array}$ & Compound name & $\begin{array}{l}\text { Molecular } \\
\text { formula }\end{array}$ & $\begin{array}{c}\text { Molecular } \\
\text { weight }\end{array}$ & $\begin{array}{c}\text { Relative } \\
\text { percentage }(\%)\end{array}$ \\
\hline 1 & 8.398 & 2-Pinene & $\mathrm{C}_{10} \mathrm{H}_{16}$ & 136.125 & 1.175 \\
\hline 2 & 9.175 & Camphene & $\mathrm{C}_{10} \mathrm{H}_{16}$ & 136.125 & 0.404 \\
\hline 3 & 10.609 & $\beta$-Pinene & $\mathrm{C}_{10} \mathrm{H}_{16}$ & 136.125 & 1.497 \\
\hline 4 & 11.008 & Myrcene & $\mathrm{C}_{10} \mathrm{H}_{16}$ & 136.125 & 0.331 \\
\hline 5 & 12.119 & 3-decene & $\mathrm{C}_{10} \mathrm{H}_{16}$ & 136.125 & 5.583 \\
\hline 6 & 12.764 & 4-isopropyltoluene & $\mathrm{C}_{10} \mathrm{H}_{14}$ & 134.110 & 1.544 \\
\hline 7 & 12.942 & D-decadiene & $\mathrm{C}_{10} \mathrm{H}_{16}$ & 136.125 & 0.628 \\
\hline 8 & 13.097 & Eucalyptol & $\mathrm{C}_{10} \mathrm{H}_{18} \mathrm{O}$ & 154.136 & 1.605 \\
\hline 9 & 14.041 & Terpinene & $\mathrm{C}_{10} \mathrm{H}_{16}$ & 136.125 & 0.870 \\
\hline 10 & 14.764 & Isobutene & $\mathrm{C}_{10} \mathrm{H}_{16}$ & 136.125 & 0.537 \\
\hline 11 & 14.875 & Terpinolene & $\mathrm{C}_{10} \mathrm{H}_{16}$ & 136.125 & 0.363 \\
\hline 12 & 16.886 & Eugenone & $\mathrm{C}_{10} \mathrm{H}_{14} \mathrm{O}$ & 150.105 & 6.896 \\
\hline 13 & 17.419 & 2-nonanol & $\mathrm{C}_{10} \mathrm{H}_{18} \mathrm{O}$ & 154.136 & 1.337 \\
\hline 14 & 17.641 & 4-nonenol & $\mathrm{C}_{10} \mathrm{H}_{18} \mathrm{O}$ & 154.136 & 0.723 \\
\hline 15 & 17.797 & Methyl isopropyl alcohol & $\mathrm{C}_{10} \mathrm{H}_{14} \mathrm{O}$ & 150.105 & 0.239 \\
\hline 16 & 18.152 & 4-allyl anisole & $\mathrm{C}_{10} \mathrm{H}_{12} \mathrm{O}$ & 148.089 & 0.737 \\
\hline 17 & 18.374 & Cis-sterol & $\mathrm{C}_{10} \mathrm{H}_{16} \mathrm{O}$ & 152.120 & 0.401 \\
\hline 18 & 19.185 & 2-isopropyl-5-methyl anisole & $\mathrm{C}_{11} \mathrm{H}_{16} \mathrm{O}$ & 164.120 & 0.306 \\
\hline 19 & 20.696 & 3,5-dimethoxytoluene & $\mathrm{C}_{9} \mathrm{H}_{12} \mathrm{O}_{2}$ & 152.084 & 11.595 \\
\hline 20 & 21.596 & Safrole & $\mathrm{C}_{10} \mathrm{H}_{10} \mathrm{O}_{2}$ & 162.068 & 17.759 \\
\hline 21 & 24.307 & Methyl eugenol & $\mathrm{C}_{11} \mathrm{H}_{14} \mathrm{O}_{2}$ & 178.099 & 12.017 \\
\hline 22 & 24.501 & 3,4,5-trimethoxytoluene & $\mathrm{C}_{10} \mathrm{H}_{14} \mathrm{O}_{3}$ & 182.094 & 4.142 \\
\hline 23 & 26.907 & Myristyl ether & $\mathrm{C}_{11} \mathrm{H}_{12} \mathrm{O}_{3}$ & 192.079 & 6.463 \\
\hline 24 & 27.918 & 3,4-propiophenone & $\mathrm{C}_{10} \mathrm{H}_{10} \mathrm{O}_{3}$ & 178.063 & 0.970 \\
\hline 25 & 28.029 & Elemene & $\mathrm{C}_{12} \mathrm{H}_{16} \mathrm{O}_{3}$ & 208.110 & 0.537 \\
\hline 26 & 30.262 & Carcinol & $\mathrm{C}_{10} \mathrm{H}_{10} \mathrm{O}_{4}$ & 194.058 & 1.392 \\
\hline 27 & 31.128 & Patchouli alcohol & $\mathrm{C}_{15} \mathrm{H}_{26} \mathrm{O}$ & 222.198 & 0.290 \\
\hline
\end{tabular}

\section{Results}

Volatile components associated with toxicity in asarum. In total, 27 volatile components were detected from asarum (Table I). The major volatile compounds identified in asarum samples were safrole (17.759\%), methyl eugenol $(12.017 \%)$, 3,5-dimethoxytoluene (11.595\%), myristyl ether $(6.463 \%)$ and benzene derivatives. These volatile components may be associated with the toxicity of asarum.

Increased organcoefficientandliverfunction in asarum-treated rats. In the third week following asarum administration, rats in the asarum group became languid, where the periphery of the eyes and auricles appeared cyanotic and the fur appeared messy and glossy. Organ coefficients and parameters of liver function, including ALT, AST and TBil, were significantly higher in the asarum-treated rats compared with those in the control group (Table II). In general, the organ coefficient and liver function were observed to be relatively consistent between animals under normal circumstances. However, in the present study, the increase in organ coefficient suggested that congestion, edema or hypertrophy of the liver occurred following asarum treatment. In addition, increases in TBil, AST and ALT suggested liver injury after asarum treatment.

Liver histology following asarum treatment. The liver tissues obtained from the control group presented with normal cellularity and well-preserved hepatic lobule, hepatic sinusoid and central vein structures (Fig. 1A). By contrast, the liver tissues from the asarum group presented with morphological tissue degeneration, including ballooning degeneration, loose cytoplasm and signs of necrosis (Fig. 1B). As observed in Fig. 1C, the histopathology score in the asarum group was significantly higher compared with that in the control group, strongly suggesting hepatotoxicity following asarum treatment.

Transcriptomics analysis of DEGs following asarum treatment. The heatmap indicated significant changes in 434 genes in liver tissues following asarum treatment, including 214 upregulated and 220 downregulated genes (Fig. 2). In addition, the KEGG biological pathway database was used to analyze the biological pathways of the DEGs. In total, 18 KEGG pathways were identified to be significantly enriched in the DEG analysis, including circadian rhythm, p53 signaling, metabolic 
Table II. Organ coefficients and parameters of liver function.

A, Organ coefficients $(\mathrm{g} / 100 \mathrm{~g} ; \mathrm{n}=7)$

\begin{tabular}{lcc}
\hline Organ & Control & Asarum \\
\hline Liver & $3.65 \pm 0.39$ & $4.23 \pm 0.30^{\mathrm{a}}$ \\
\hline
\end{tabular}

B, Liver function $(\mathrm{n}=5)$

\begin{tabular}{lcc}
\hline Parameter & Control & Asarum \\
\hline TBil & $2.66 \pm 0.39$ & $29.2 \pm 3.65^{\mathrm{a}}$ \\
ALT & $46.2 \pm 2.71$ & $64.0 \pm 5.58^{\mathrm{a}}$ \\
AST & $40.0 \pm 9.87$ & $77.2 \pm 16.8^{\mathrm{a}}$ \\
\hline
\end{tabular}

Values are expressed as the mean \pm standard deviation of at least three independent experiments. ${ }^{\mathrm{a}} \mathrm{P}<0.05$ vs. control following unpaired t-test with Welch's correction. AST, aspartate transferase; TBil, total bilirubin; ALT, alanine transaminase.

pathways, steroid biosynthesis and bile secretion pathways (Table III). GO analysis was subsequently performed and it was observed that the functional terms 'cellular process' and 'metabolic processes' accounted for the largest percentages in the protein analysis through evolutionary relationships (PATHER) GO-Slim Biological Process category (Fig. 3A), 'binding' and 'catalytic activity' were the highest in the PATHER GO-Slim Molecular Function category (Fig. 3B), 'cell' and 'organelle' occupied the largest proportion in the PATHER GO-Slim Cellular Component category (Fig. 3C) and finally, 'hydrolase' and 'transcription factor' accounted for the highest percentage in the PATHER Protein Class (Fig. 3D). The numbers provided by a GO slim are an annotation count not a gene product count, and so gene products may be present in more than one bin.

Identification and analysis of metabolites following asarum treatment. The total ion chromatograms (Fig. 4) demonstrated marked differences in the metabolite profiles between the control and asarum groups, which were greater using multivariate analysis methods. PCA is able to convert multiple indicators into a number of comprehensive indicators to analyze differences between groups, whereas PLS-DA is able to enhance the separation between groups. A distinct separation was observed in the PLS-DA (Fig. 5A) and PCA score plots (Fig. 5B) of the two groups, where the R2 and Q2 were indicated to be 0.91 and 0.81 , respectively, suggesting that liver metabolism was altered following asarum treatment.

Relative intensity analysis is frequently used to investigate changes in the levels of biomarkers (27). In total, 14 metabolites were identified as biomarkers from liver homogenates (Table IV). These 14 metabolites identified are represented by the peaks in Fig. 4. Compared with the control group, the levels of L-alanine, taurine, phosphoglycolic acid, xylitol, D-sorbitol, L-lactic acid, myo-inositol and L-aspartic acid were markedly increased, whilst the levels of spermidine, L-methionine, maltotriose, trehalose, ribitol and L-proline were indicated to be markedly lower in the asarum group (Fig. 6).
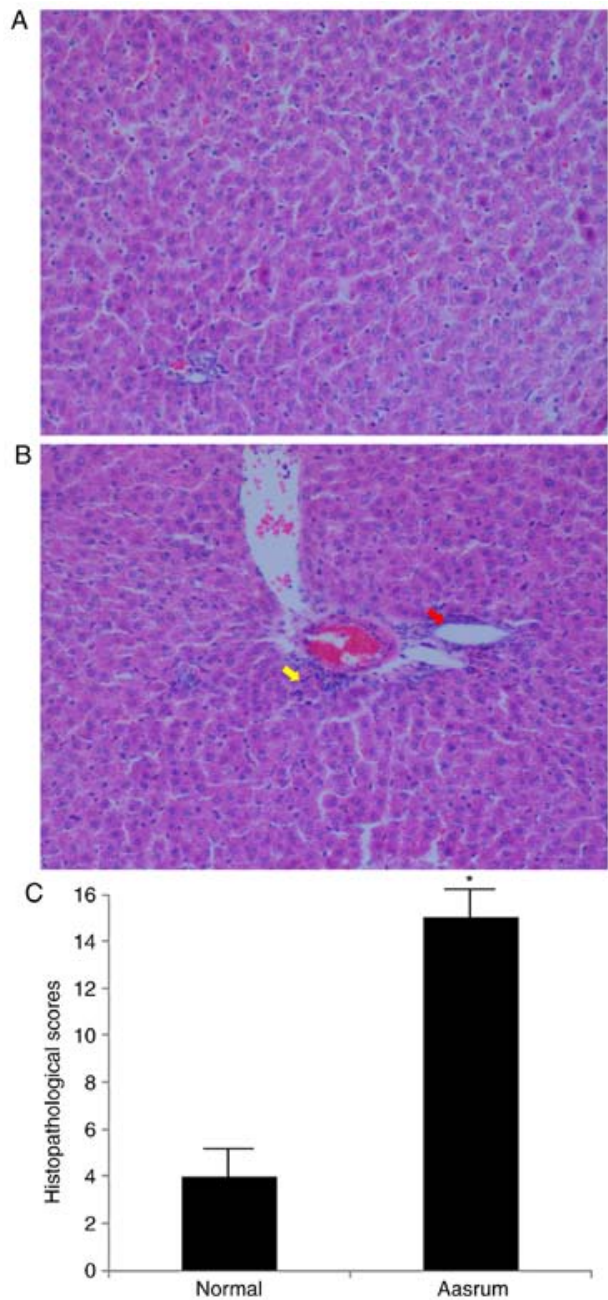

Figure 1. Asarum treatment-induced hepatotoxicity. Hematoxylin and eosin staining of pathological liver tissues isolated from rats in (A) the normal group and (B) the Asarum group (magnification, x200). Cell necrosis (yellow arrow) and ballooning degeneration (red arrow) were observed. (C) Quantified histopathological scores. Values are expressed as the mean \pm standard deviation. ${ }^{*} \mathrm{P}<0.05$ vs. Control group.

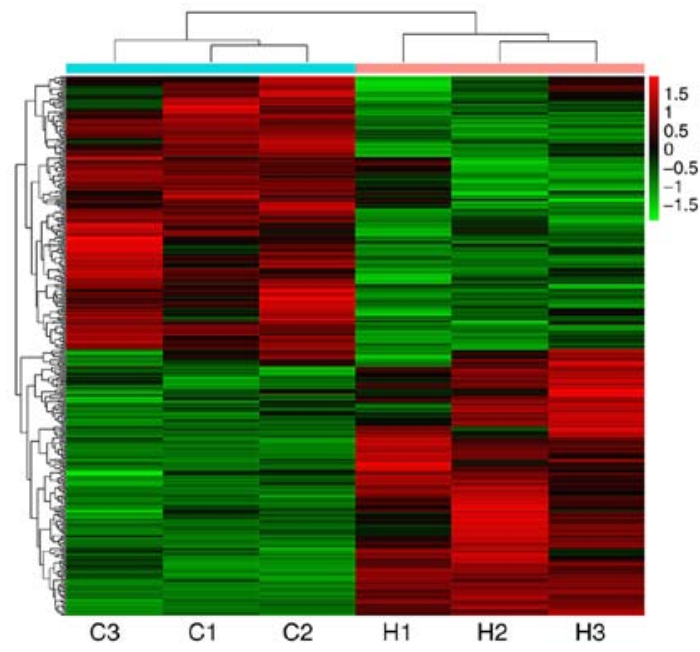

Figure 2. Heatmap of differentially expressed genes in each liver isolated from rats in the control and Asarum groups. Following asarum treatment, 434 genes were significantly altered in liver tissues, including 214 upregulated and 220 downregulated genes. The horizontal coordinate displays the groups and the vertical coordinate the genes. Red indicates upregulated and green downregulated genes. $\mathrm{C}$, control; $\mathrm{H}$, asarum 
Table III. Significantly enriched pathways based on KEGG pathway analysis.

\begin{tabular}{|c|c|c|c|c|}
\hline Pathway ID & Pathway & Upregulated_KO & Downregulated_KO & P-value \\
\hline ko04710 & Circadian rhythm & ARNT1, NPAS2 & BHLHB2, NR1D1, PER2 & $1.92 \times 10^{-8}$ \\
\hline ko04115 & p53 signaling pathway & $\begin{array}{l}\text { SESN1, CDKN1A, ZMAT3, } \\
\text { GTSE1 }\end{array}$ & SERPINE1, CCNE, CCND1 & $2.16 \times 10^{-4}$ \\
\hline ko04713 & Circadian entrainment & GRIA3, NOS1 & ADCY3, RYR1, PER2 & $7.01 \times 10^{-4}$ \\
\hline ko01100 & Metabolic pathways & ASNS, GALE, CTH, GK & AK1, GCK & $2.32 \times 10^{-3}$ \\
\hline ko00100 & Steroid biosynthesis & DHCR7 & SQLE, MESO1 & $5.65 \times 10^{-3}$ \\
\hline ko00750 & Vitamin B6 metabolism & N/A & SERC, PDXP & $1.06 \times 10^{-2}$ \\
\hline ko04976 & Bile secretion & $\mathrm{N} / \mathrm{A}$ & $\begin{array}{l}\text { CYP7A1, SULT2A1, } \\
\text { HMGCR, ABCG5 }\end{array}$ & $1.13 \times 10^{-2}$ \\
\hline ko02010 & $\mathrm{ABC}$ transporters & ABCG9, ABCG3 & ABCA4, ABCG5 & $1.16 \times 10^{-2}$ \\
\hline ko04978 & Mineral absorption & HEPH & MT1_2, ATP1A & $1.27 \times 10^{-2}$ \\
\hline ko03320 & PPAR signaling pathway & SCD, PPARD, GK & FABP4 & $2.12 \times 10^{-2}$ \\
\hline ko00660 & $\begin{array}{l}\text { C5-Branched dibasic acid } \\
\text { metabolism }\end{array}$ & N/A & IRG1 & $2.34 \times 10^{-2}$ \\
\hline ko04970 & Salivary secretion & ATP2B, NOS1 & ATP1A, ADCY3 & $4.16 \times 10^{-2}$ \\
\hline ko04972 & Pancreatic secretion & ATP2A, АTP2B & ATP1A, ADCY3, CLCA2 & $4.29 \times 10^{-2}$ \\
\hline ko00260 & $\begin{array}{l}\text { Glycine, serine and threonine } \\
\text { metabolism }\end{array}$ & $\mathrm{CTH}$ & SERC, SERA & $4.45 \times 10^{-2}$ \\
\hline ko04724 & Glutamatergic synapse & GRIA3, PLD1_2 & ADCY3, SLC1A7 & $4.48 \times 10^{-2}$ \\
\hline ko04020 & Calcium signaling pathway & ADORA2B, ATP2A & ADCY3, RYR1 & $4.61 \times 10^{-2}$ \\
\hline ko00909 & $\begin{array}{l}\text { Sesquiterpenoid and triterpenoid } \\
\text { biosynthesis }\end{array}$ & N/A & SQLE & $4.62 \times 10^{-2}$ \\
\hline ko04212 & $\begin{array}{l}\text { Longevity-regulating } \\
\text { pathway-worm }\end{array}$ & SCD & MT1_2, MAP2K6 & $4.64 \times 10^{-2}$ \\
\hline
\end{tabular}

KEGG, Kyoto Encyclopedia of Genes and Genomes; KO, KEGG identifier; ARNTl, aryl hydrocarbon receptor nuclear translocator like 1; NPAS2, neuronal PAS domain protein 2; BHLHB2, basic helix-loop-helix protein 2; NR1D1, nuclear receptor subfamily 1 group D member 1; PER2, period circadian regulator 2; SESN1, sestrin 1; CDKN1A, cyclin dependent kinase inhibitor 1A; ZMAT3, zinc finger matrin-type 3; GTSE1, G2 and S-phase expressed 1; SERPINE1, serpin family E member 1; CCNE, cyclin E; CCND1, cyclin D1; GRIA3, glutamate ionotropic receptor AMPA type subunit 3; NOS1, nitric oxide synthase 1; ADCY3, adenylate cyclase 3; RYR1, ryanodine receptor 1; ASNS, asparagine synthetase; GALE, UDP-galactose-4-epimerase; CTH, cystathionine $\gamma$-lyase; GK, glycerol kinase; AK1, adenylate kinase 1; GCK, glucokinase; DHCR7, 7-dehydrocholesterol reductase; SQLE, squalene epoxidase; MESO1, transcription factor 15; SERC, ATPase sarcoplasmic/endoplasmic reticulum Ca2+ transporting 1; PDXP, pyridoxal phosphatase; CYP7A1, cytochrome P450 family 7 subfamily A member 1; SULT2A1, sulfotransferase family 2A member 1; HMGCR, 3-hydroxy-3-methylglutaryl-CoA reductase; ABCG5, ATP binding cassette subfamily $\mathrm{G}$ member 5; ABCG9, ABC-2 type transporter family protein; ABCG3, ATP binding cassette subfamily G member 3; ABCA4, ATP binding cassette subfamily A member 4; HEPH, hephaestin; MT1_2, metallothionein 1/2; ATP1A, sodium/potassium-transporting ATPase subunit $\alpha$; SCD, stearoyl-CoA desaturase; PPARD, peroxisome proliferator activated receptor delta; FABP4, fatty acid binding protein 4; IRG1, aconitate decarboxylase 1; ATP2B, P-type Ca2+ transporter type 2B; ATP2A, P-type Ca2+ transporter type 2A; CLCA2, chloride channel accessory 2; SERA, selenate/chlorate reductase subunit $\alpha$; PLD1_2, pyridoxal 4-dehydrogenase; SLC1A7, solute carrier family 1 member 7; ADORA2B, adenosine A2b receptor; MAP2K6, mitogen-activated protein kinase kinase 6.

The profiles of the biomarkers were subsequently imported into the MetaboAnalyst online tool to explore possible metabolic pathways affected by asarum treatment. A total of six metabolic pathways were identified as important metabolic pathways, as listed in Fig. 7. Therefore, these observations suggest that asarum treatment disrupted the composition of endogenous metabolites in rats, inducing disturbances in taurine, hypotaurine and amino acid metabolism.

Integrated analysis of metabolomics and transcriptomics data. MetScape 3 may be used to visualize and interpret metabolomics and gene expression profiling data in an integrated metabolic network $(28,30)$. To investigate the correlation of metabolites and gene expression affected by asarum treatment, metabolite and gene expression profiles were imported into the Cytoscape for Metscape analysis. The metabolite-gene associated network was revealed to be mainly associated with 'bile acid biosynthesis' and 'amino acid metabolism' (Fig. 8). The differentially expressed/abundant genes and metabolites associated with these pathways are presented in Table V. The results suggested that bile acid biosynthesis and amino acid metabolism may be the key processes associated with the mechanism of asarum-mediated hepatotoxicity. Because no metabolite changes were found in the p53 signaling pathway, they were not shown in the figure during the integrated analysis. 

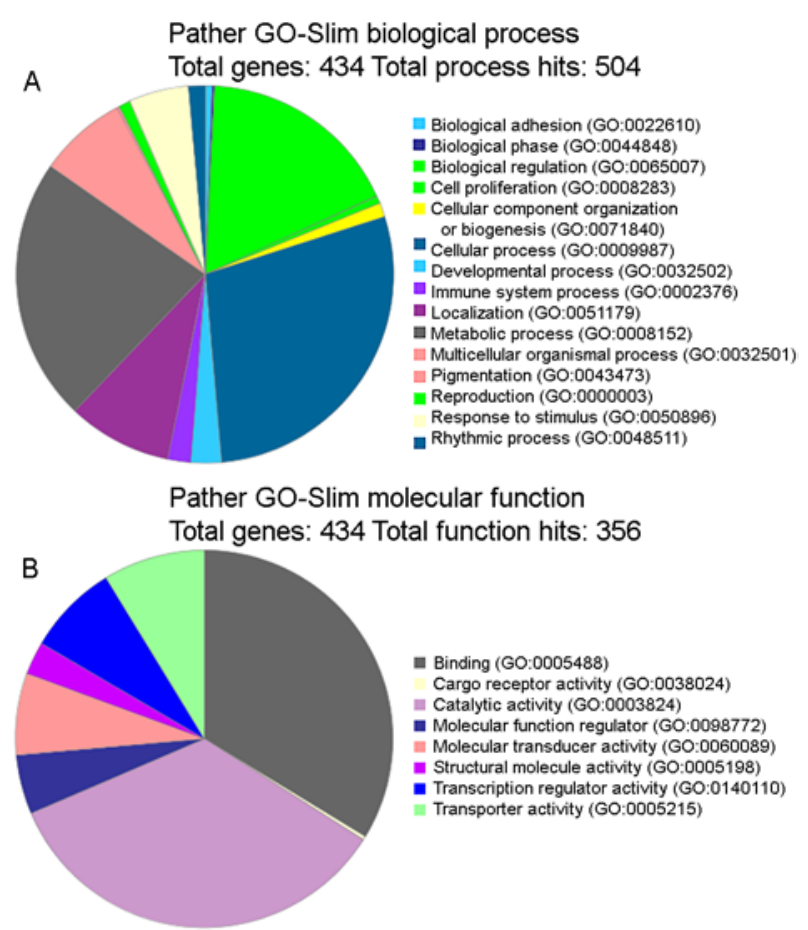

Pather GO-Slim cellular component

Total genes: 434 Total component hits: 285

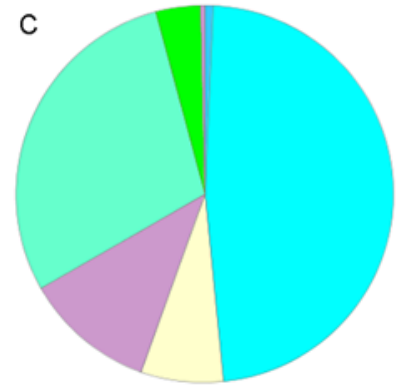

II Cell junction (GO:0030054

Cell (GO:0005623)

Extracellular region (GO:0005576)

Membrane (GO:0016020)

Organelle (GO:0043226)

Protein-containing complex (GO:0032991)

= Supramolecular complex (GO:0099080)

Pather protein class

Total genes: 434 Total protein hits: 285

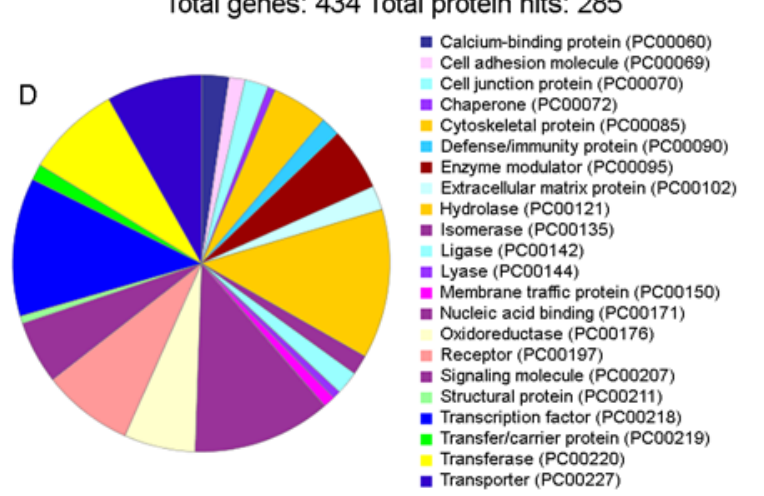

Figure 3. Gene Ontology analysis of differentially expressed genes associated with asarum treatment. (A) PATHER GO-Slim Biological Process category. (B) PATHER GO-Slim Molecular Function category. (C) PATHER GO-Slim Cellular Component category. (D) PATHER Protein Class. GO, gene ontology; PATHER, protein analysis through evolutionary relationships.

\section{Discussion}

Toxicity studies are a vital integral component of TCM development (31). In the present study, the asarum group exhibited significantly enhanced organ coefficients and liver function compared with the control group. In addition, pathological liver tissue staining indicated significant liver
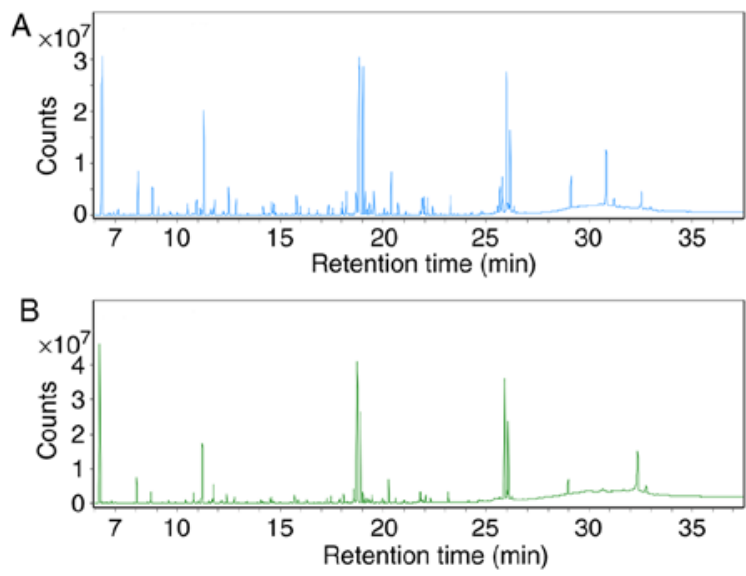

Figure 4. Total ion chromatogram of metabolites obtained from liver tissues isolated from rats in the Control and Asarum groups. (A) Control group and (B) asarum treatment group. The $\mathrm{x}$-axis displays the retention time and the $\mathrm{y}$-axis the peak abundance.
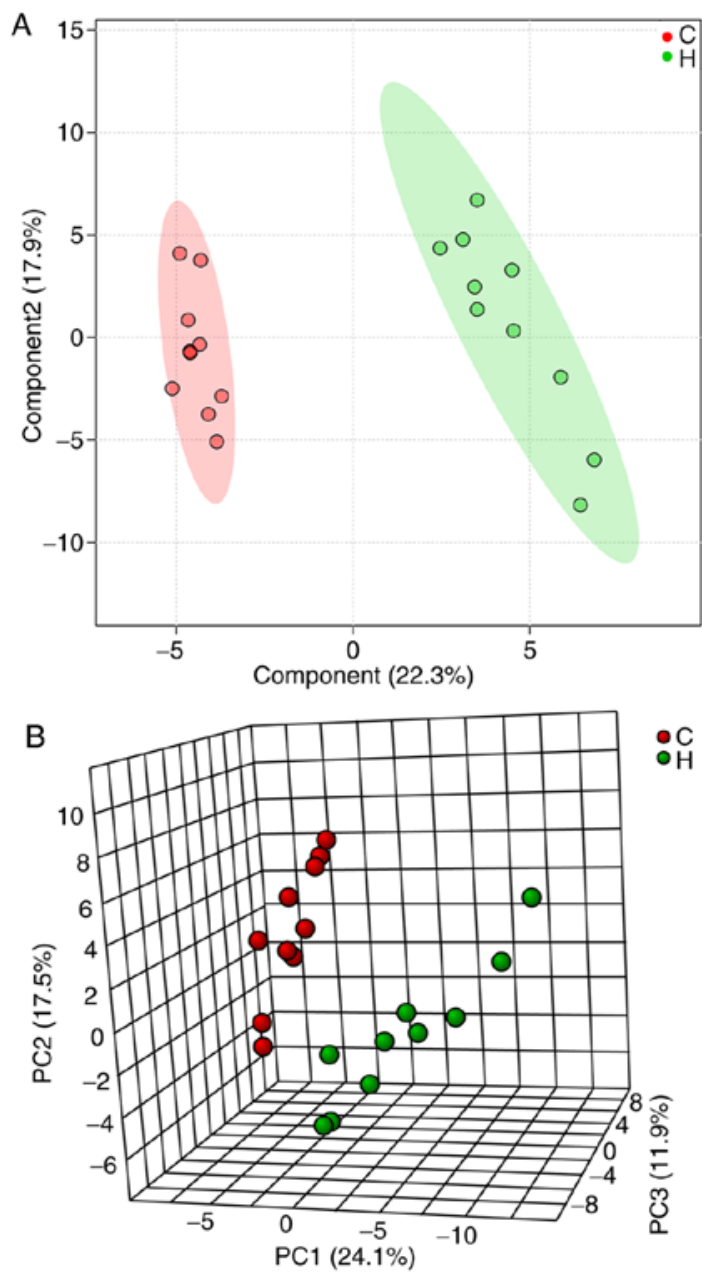

Figure 5. Metabolic profiling of liver tissues isolated from rats in the control and asarum groups. (A) PC analysis and (B) partial least squares-discriminant analysis score plots, which indicated a distinct separation between the control and asarum groups. $\mathrm{C}$, control; $\mathrm{H}$, asarum; PC, principal component.

damage following asarum treatment, further supporting the notion of asarum-induced hepatotoxicity. Transcriptomics and metabolomics techniques were subsequently performed 
Table IV. Identification of potential biomarkers.

\begin{tabular}{ccllll}
\hline No. & Retention time (min) & \multicolumn{1}{c}{ Metabolite } & P-value & Fold change & \multicolumn{1}{c}{ Associated pathway } \\
\hline 1 & 8.0415 & L-lactic acid & $2.40 \times 10^{-5}$ & 6.128 & Glycolysis \\
2 & 8.7148 & L-alanine & $3.75 \times 10^{-4}$ & 2.832 & Alanine, aspartate and glutamate metabolism \\
3 & 9.8959 & L-proline & $3.60 \times 10^{-3}$ & 3.598 & Arginine and proline metabolism \\
4 & 13.2286 & L-methionine & $4.24 \times 10^{-3}$ & 2.756 & Cysteine and methionine metabolism \\
5 & 13.3784 & L-aspartic acid & $4.04 \times 10^{-3}$ & 5.324 & Alanine, aspartate and glutamate metabolism \\
6 & 15.4022 & Phosphoglycolic acid & $2.42 \times 10^{-3}$ & 2.514 & Glyoxylate and dicarboxylate metabolism \\
7 & 15.5519 & Taurine & $8.68 \times 10^{-3}$ & 2.406 & Primary bile acid biosynthesis \\
8 & 16.8073 & Ribitol & $3.00 \times 10^{-6}$ & 7.887 & Pentose and glucuronate interconversions \\
9 & 16.8572 & Xylitol & $2.83 \times 10^{-4}$ & 3.124 & Pentose and glucuronate interconversions \\
10 & 18.2734 & D-sorbitol & $8.15 \times 10^{-4}$ & 2.376 & Fructose and mannose metabolism \\
11 & 20.6283 & Myo-inositol & $7.22 \times 10^{-3}$ & 3.198 & Galactose metabolism \\
12 & 22.1431 & Spermidine & $1.03 \times 10^{-3}$ & 3.054 & Arginine and proline metabolism \\
13 & 26.0201 & Trehalose & $4.26 \times 10^{-3}$ & 12.26 & Starch and sucrose metabolism \\
14 & 30.6569 & Maltotriose & $5.95 \times 10^{-4}$ & 33.94 & Carbohydrate digestion and absorption \\
\hline
\end{tabular}

Table V. Metabolic pathways identified in the interactome network using the Cytoscape software 3.6.1, incorporating gene transcription and metabolite profiles.

\begin{tabular}{lll}
$\begin{array}{l}\text { Metabolic pathways enriched } \\
\text { within the interactome network }\end{array}$ & \multicolumn{1}{c}{$\begin{array}{c}\text { Differentially } \\
\text { expressed genes }\end{array}$} & $\begin{array}{c}\text { cesped metabolites } \\
\text { expressed }\end{array}$ \\
\hline $\begin{array}{l}\text { Bile acid synthesis } \\
\text { Amino acid metabolism }\end{array}$ & CYP7A1, SULT2A1, HMGCR & Taurine, L-aspartic acid \\
& ASNS, CTH, DNMT3B, SAT1 & L-alanine, S-lactic acid, spermidine, \\
p53 signaling pathway & SESN1, CDKN1A, ZMAT3, GTSE1, & None
\end{tabular}

CYP7A1, cholesterol 7 $\alpha$-hydroxylase; SULT2A1, bile salt sulfotransferase; HMCGR, 3-hydroxy-3-methylglutaryl-CoA reductase; ASNS, asparagine synthetase; CTH, cystathionine $\gamma$-lyase; DNMT3B, DNA methyltransferase $3 \beta$; SAT1, spermidine/spermine N1-acetyltransferase 1; SESN1, sestrin 1; CDKN1A, cyclin dependent kinase inhibitor 1A; ZMAT3, zinc finger matrin-type 3; GTSE1, G2 and S-phase expressed 1; SERPINE1, serpin family E member 1; CCNE, cyclin E; CCND1, cyclin D1.

to investigate the potential mechanism of asarum-mediated hepatotoxicity. The transcription profiles exhibited by liver tissues were first studied, where P53 signaling, metabolic pathways, steroid biosynthesis and bile secretion pathways were indicated to be the most relevant signaling pathways associated with asarum treatment. The metabolomic profiles of liver tissues from the control and asarum groups were next analyzed, which revealed that the associated metabolites are mainly members of the taurine, hypotaurine and amino acid metabolic pathways. The obtained transcriptomics and metabolomics datasets were next integrated and the data indicated that asarum-induced hepatotoxicity was mainly mediated through alterations of bile acid biosynthesis and amino acid metabolism.

Bile acids, synthesized from cholesterol in the liver, serve an important role in the regulation of energy, glucose and lipid metabolism (32). Cholesterol 7 $\alpha$-hydroxylase (CYP7A1) serves as the rate-limiting enzyme in the classical biogenic pathway of bile acid synthesis (33), the activity of which restricts the synthesis of bile acids in the body. By regulating CYP7A1 activity, the synthesis of bile acids may be steadily maintained. During bile acid secretion, bile acids may conjugate with either taurine or glycine, a process that lowers their $\mathrm{pKa}$ and increases their solubility, resulting in their secretion into the intestines with bile in a process that is catalyzed by bile salt sulfotransferase (SULT2A1) (34). In the present study, CYP7A1 and SULT2A1 were indicated to be markedly downregulated following asarum administration, whilst the level of taurine was markedly increased. In addition, the levels of TBil were increased, suggesting that the synthesis and secretion of bile acids were affected by asarum administration.

The liver is the principal organ of amino acid metabolism. Under physiological conditions, basal levels of amino acid metabolism are high due to the high abundance of associated enzymes. Consequently, liver damage frequently results in aberrant amino acid metabolism and eventually loss of liver function. Three amino acids function as important 

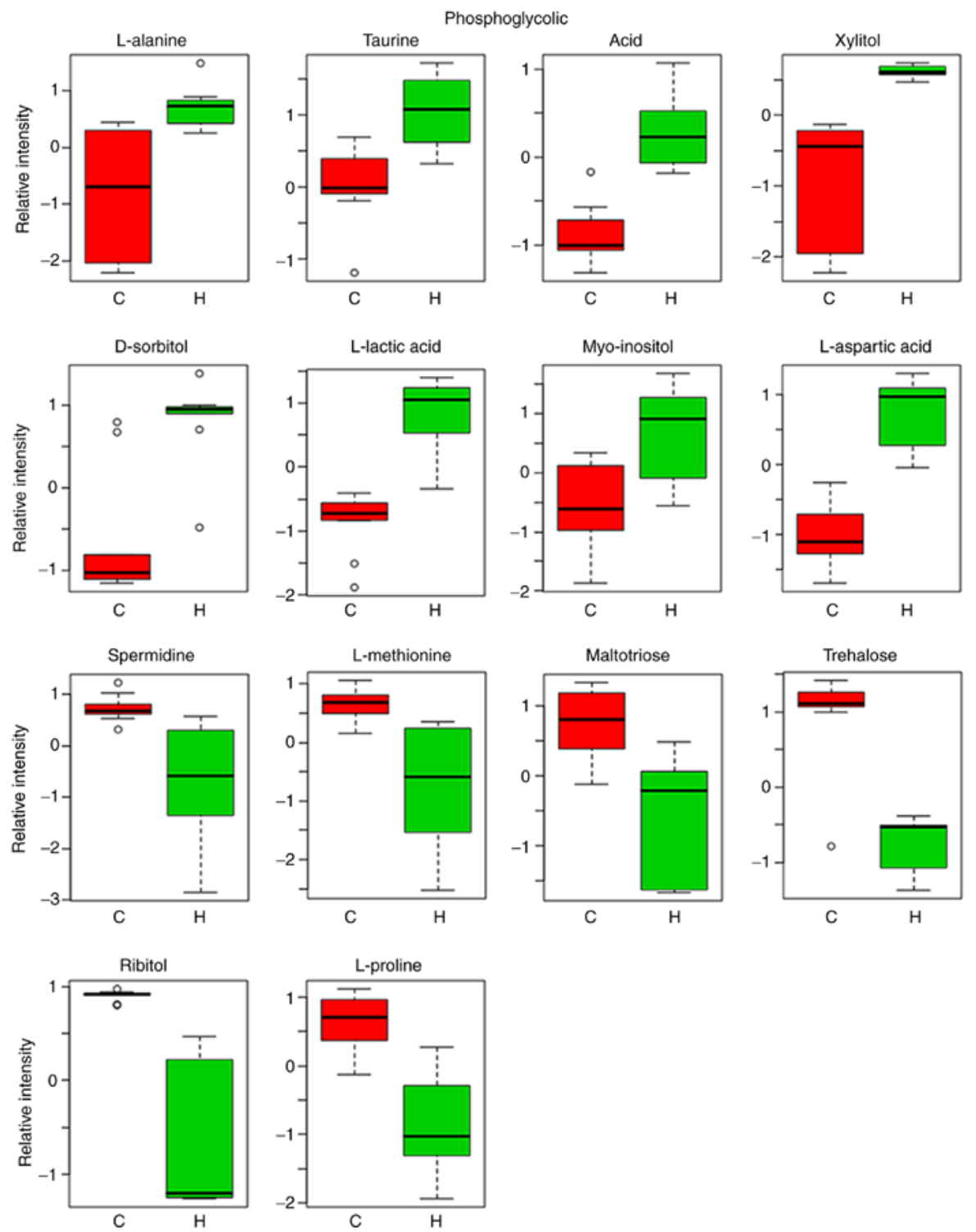

Figure 6. Comparison of relative intensities of biomarkers observed in the liver tissues isolated from rats in the control and asarum groups. Following asarum treatment, 14 metabolites were indicated to be significantly altered in liver tissues, including 8 upregulated and 6 downregulated metabolites. The y-axis displays the relative intensity and the $\mathrm{x}$-axis the experimental groups. $\mathrm{C}$, control; $\mathrm{H}$, asarum. The upper and lower horizontal lines represent the maximum and minimum values, the upper and lower borders of the box represent the upper and lower quartiles, the bars represent the median, and the circles represent the outliers.

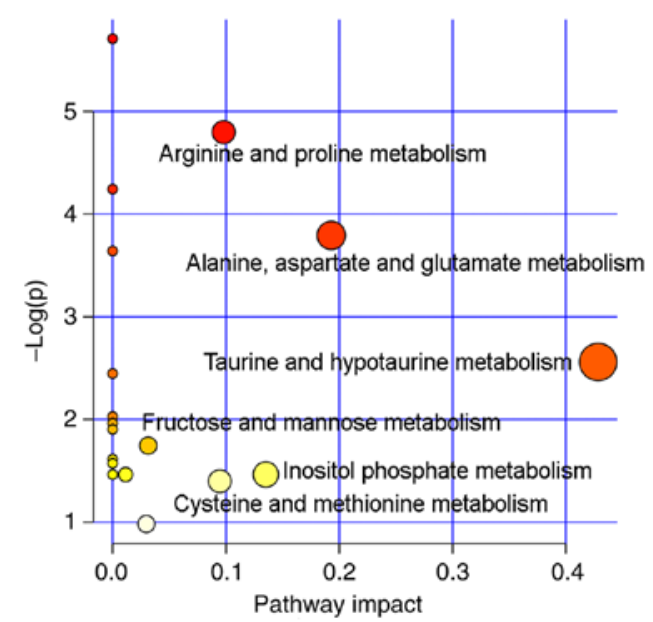

Figure 7. Metabolite enrichment analysis as a result of asarum treatment in the liver, where six metabolic pathways were identified to be the most important metabolic pathways. intermediates in energy metabolism (35): Alanine is involved in the alanine-glucose cycle in the body; aspartic acid serves as the bridge between amino acid and sugar metabolism, as it reacts with pyridoxal phosphates to form oxaloacetate in a reaction catalyzed by transaminases to produce sugar by gluconeogenesis, whilst proline is synthesized by the glutamate pathway. In addition, taurine may be synthesized from methionine. The covalent interaction between taurine and bile acid enhances the solubility of bile acid, facilitating the exclusion from extrahepatic cells and consequently reduction of bile acid levels in the liver (36). In the present study, it was indicated that the levels L-alanine and L-aspartic acid were markedly incrased following asarum administration, whilst those of L-proline and L-methionine were markedly reduced. This observation, together with the result that the activities of AST and ALT were also increased, further suggests that asarum treatment induced abnormalities in amino acid metabolism. 

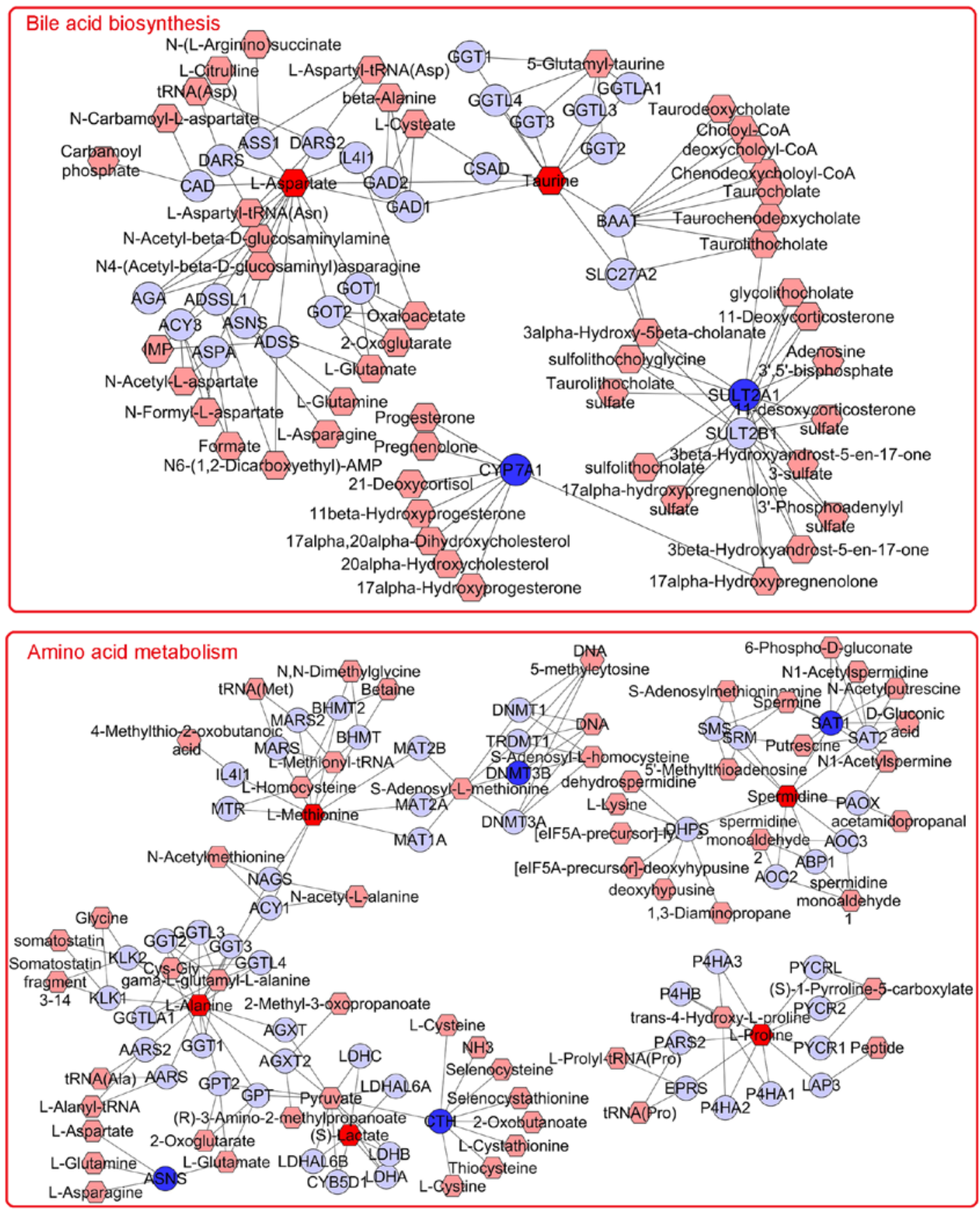

Figure 8. Networks of the metabolites and genes were visualized using Metscape 3. The entries were obtained from an integrated analysis of the asarum-mediated hepatotoxicity mechanism by combining metabolomics and transcriptomics data. The metabolite-gene associated network was mainly associated with bile acid biosynthesis and amino acid metabolism. Hexagons indicate metabolites, circles represent genes, edges indicate the association between each node. Input genes are shown in dark blue, input metabolites are shown in dark red. Light colors indicate differentially expressed genes. CAD, carbamoyl-phosphate synthetase 2 aspartate transcarbamylase and dihydroorotase; DARS, aspartyl-tRNA synthetase; ASS1, argininosuccinate synthase 1; DARS2, aspartyl-tRNA synthetase 2; IL4I1, interleukin 4 induced 1; GAD2, glutamate decarboxylase 2; GAD1, glutamate decarboxylase 1; CSAD, cysteine sulfinic acid decarboxylase; GGT1, $\gamma$-glutamyltransferase 1; GGTL4, $\gamma$-glutamyltransferase light chain 2; GGT3, $\gamma$-glutamyl transpeptidase 3; GGTL3, $\gamma$-glutamyltransferase 7; GGTLA1, $\gamma$-glutamyltransferase 5; GGT2, $\gamma$-glutamyltransferase 2; BAAT, bile acid-CoA, amino acid N-acyltransferase; SLC27A2, solute carrier family 27 member 2; AGA, aspartylglucosaminidase; ACY3, aminoacylase 3; ADSSL1 adenylosuccinate synthase 1; ASNS, asparagine synthetase; ADSS, adenylosuccinate synthase 2; GOT2, glutamic-oxaloacetic transaminase 2; GOT1, glutamic-oxaloacetic transaminase 1; SULT2B1, sulfotransferase family 2B member 1; MTR, 5-methyltetrahydrofolate-homocysteine methyltransferase; MARS, methionyl-tRNA synthetase 1; MARS2, methionyl-tRNA synthetase 2; BHMT2, betaine-homocysteine S-methyltransferase 2; BHMT, betaine-homocysteine S-methyltransferase; MAT2B, methionine adenosyltransferase 2B; MAT2A, methionine adenosyltransferase 2A; MAT1A, methionine adenosyltransferase 1A; DNMT1, DNA methyltransferase 1; TRDMT1, tRNA aspartic acid methyltransferase 1; DNMT3B, DNA methyltransferase $3 \beta$; DNMT3A, DNA methyltransferase 3; SMS, spermine synthase; SRM, spermidine synthase; SAT1, spermidine/spermine N1-acetyltransferase 1; SAT2, spermidine/spermine N1-acetyltransferase family member 2; PAOX, polyamine oxidase; DHPS, deoxyhypusine synthase; AOC2, amine oxidase copper containing 2; ABP1, actin-binding protein 1; AOC3, amine oxidase copper containing 3; KLK1, kallikrein 1; KLK2, kallikrein 2; AARS2, alanyl-tRNA synthetase 2; AARS, alanyl-tRNA synthetase; GPT2, glutamic-pyruvic transaminase 2; GPT, glutamic-pyruvic transaminase; AGXT, alanine-glyoxylate and serine-pyruvate aminotransferase; AGXT2, alanine-glyoxylate aminotransferase 2; LDHC, lactate dehydrogenase C; LDHAL6A, lactate dehydrogenase A like 6A; LDHAL6B, lactate dehydrogenase A like 6B; CYB5D1, cytochrome b5 domain containing 1; LDHB, lactate dehydrogenase B; LDHA, lactate dehydrogenase A; CTH, cystathionine $\gamma$-lyase; P4HA3, prolyl 4-hydroxylase subunit $\alpha 3$; P4HB, prolyl 4-hydroxylase subunit $\beta$; PARS2, prolyl-tRNA synthetase 2; EPRS, glutamyl-prolyl-tRNA synthetase 1; P4HA2, prolyl 4-hydroxylase subunit $\alpha 2$; P4HA1, prolyl 4-hydroxylase subunit $\alpha 1$; PYCRL, pyrroline-5-carboxylate reductase 3; PYCR2, pyrroline-5-carboxylate reductase 2; PYCR1, pyrroline-5-carboxylate reductase 1; LAP3, leucine aminopeptidase 3. 
The transcriptomic pathway enrichment analysis of the present study also indicated that the activity of the P53 signaling pathway was increased by asarum treatment. P53 signaling may induce apoptosis through a number of different pathways in a manner that is highly dependent on the cell type and upstream signaling (37). This may be associated with the presence of carcinogenic chemicals, e.g. safrole in asarum. A recent study reported that P53 signaling promoted bile acid disposition and alleviated cholestastic syndrome (38). However, further study is required to validate the role of the P53 signaling pathway, in addition to the role of each individual component of asarum, on hepatotoxicity.

In conclusion, the present study demonstrated that asarum-induced hepatoxicity may be mediated through the disruption of the bile acid and amino acid metabolism, in addition to the P53 signaling pathway. These data may provide novel insight into the mechanism of the hepatotoxic effect of asarum, which may aid in the development of novel clinical diagnostic strategies and therapeutic interventions for asarum poisoning.

\section{Acknowledgements}

Not applicable.

\section{Funding}

This work was supported by the National Natural Science Foundation of China (grant no. 81573625) and the Science and Technology Innovation Team Project of Hubei Provincial Department of Education for Young and Middle-aged scientists (grant no. T201608).

\section{Availability of data and materials}

The datasets used and/or analyzed during the present study are available from the corresponding author on reasonable request.

\section{Authors' contributions}

SC and LTH collected and analyzed the data and prepared the manuscript; YML, SQY and SHH collected the data. SSM, WQD, and JJL analysed the data and critically revised the content of this manuscript. ZXZ, QW and FH made substantial contributions to the conception of the study and to the manuscript. All authors read and approved the final manuscript.

\section{Ethics approval and consent to participate}

The present study was approved by the animal ethics committee of Hubei University of TCM (Wuhan, China; approval no. HUCMS201903001).

\section{Patient consent for publication}

Not applicable.

\section{Competing interests}

The authors declare that they have no competing interests.

\section{References}

1. Jing Y, Zhang YF, Shang MY, Liu GX, Li YL, Wang X and Cai SQ: Chemical constituents from the roots and rhizomes of Asarum heterotropoides var. mandshuricum and the in vitro anti-inflammatory activity. Molecules 22: pii: E125, 2017.

2. Zhang W, Zhang J, Zhang $M$ and Nie L: Protective effect of Asarum extract in rats with adjuvant arthritis. Exp Ther Med 8: 1638-1642, 2014.

3. Han S, Huang J, Hou J and Wang S: Screening epidermal growth factor receptor antagonists from Radix et Rhizoma Asari by two-dimensional liquid chromatography. J Sep Sci 37: 1525-1532, 2014.

4. Huang J, Wang HQ, Zhang C, Li GY, Lin RC and Wang JH: A new tetrahydrofuran-type lignan with anti-inflammatory activity from Asarum heterotropoides Fr. Schmidt var. mandshuricum. J Asian Nat Prod Res 16: 387-392, 2014.

5. Tian HY, Hu J and Wang L: Controlled observation of non-blister acupoint sticking and electroacupuncture for bronchial asthma. Zhongguo Zhen Jiu 33: 485-489, 2013 (In Chinese).

6. Kim JR, Perumalsamy H, Lee JH, Ahn YJ, Lee YS and Lee SG: Acaricidal activity of Asarum heterotropoides root-derived compounds and hydrodistillate constitutes toward Dermanyssus gallinae (Mesostigmata: Dermanyssidae). Exp Appl Acarol 68: 485-495, 2016.

7. Cartus AT, Stegmüller S, Simson N, Wahl A, Neef S, Kelm H and Schrenk D: Hepatic metabolism of carcinogenic $\beta$-asarone. Chem Res Toxicol 28: 1760-1773, 2015.

8. Wang Y, Guo S, Cao J, Pang X, Zhang Z, Chen Z, Zhou Y, Geng Z, Sang Y and Du S: Toxic and Repellent Effects of Volatile Phenylpropenes from Asarum heterotropoides on Lasioderma serricorne and Liposcelis bostrychophila. Molecules 23: pii: E2131, 2018.

9. Yang B, Xie Y, Guo M, Rosner MH, Yang H and Ronco C: Nephrotoxicity and Chinese herbal medicine. Clin J Am Soc Nephrol 13: 1605-1611, 2018.

10. Coghlan ML, Haile J, Houston J, Murray DC, White NE, Moolhuijzen P, Bellgard MI and Bunce M: Deep sequencing of plant and animal DNA contained within traditional Chinese medicines reveals legality issues and health safety concerns. PLoS Genet 8: e1002657, 2012.

11. Li Y, Han L, Huang C, Dai W, Tian G, Huang F, Li J, Liu J, Wang $Q$ and Zhou Z: New contributions to asarum powder on immunology related toxicity effects in lung. Evid Based Complement Alternat Med 2018: 1054032, 2018.

12. Li XW, Morinaga O, Tian M, Uto T, Yu J, Shang MY, Wang X, Cai SQ and Shoyama Y: Development of an Eastern blotting technique for the visual detection of aristolochic acids in Aristolochia and Asarum species by using a monoclonal antibody against aristolochic acids I and II. Phytochem Anal 24: 645-653, 2013.

13. Hu L, Shao H, He J, Zhong L, Song Y and Wu F: Cytotoxicity of safrole in HepaRG cells: Studies on the role of CYP1A2-mediated ortho-quinone metabolic activation. Xenobiotica 49: 1504-1515, 2019.

14. Yang AH, Zhang L, Zhi DX, Liu WL, Gao X and He X: Identification and analysis of the reactive metabolites related to the hepatotoxicity of safrole. Xenobiotica 48: 1164-1172, 2018.

15. Patel DN, Ho HK, Tan LL, Tan MM, Zhang Q, Low MY, Chan CL and Koh HL: Hepatotoxic potential of asarones: In vitro evaluation of hepatotoxicity and quantitative determination in herbal products. Front Pharmacol 6: 25, 2015.

16. Song J, Zhong RL, Xia Z, Wu H, Zhong QX, Zhang ZH, Wei YJ, Shi ZQ, Feng L and Jia XB: Research and application of hepatotoxicity evaluation technique of traditional Chinese medicine. Zhongguo Zhong Yao Za Zhi 42: 41-48, 2017.

17. Ye H, Nelson LJ, Gómez Del Moral M, Martinez-Naves E and CuberoFJ: Dissecting the molecularpathophysiology of drug-induced liver injury. World J Gastroenterol 24: 1373-1385, 2018.

18. Xin J, Zhang RC, Wang L and Zhang YQ: Researches on transcriptome sequencing in the study of traditional Chinese medicine. Evid Based Complement Alternat Med 2017: 7521363, 2017.

19. Jin J, Kang W, Zhong C, Qin Y, Zhou R, Liu H, Xie J, Chen L, Qin Y and Zhang S: The pharmacological properties of Ophiocordyceps xuefengensis revealed by transcriptome analysis. J Ethnopharmacol 219: 195-201, 2018.

20. Misra BB, Upadhayay RP, Cox LA and Olivier M: Optimized GC-MS metabolomics for the analysis of kidney tissue metabolites. Metabolomics 14: 75, 2018. 
21. Zhang Y, Li H, Hu T, Li H, Jin G and Zhang Y: Metabonomic profiling in study hepatoprotective effect of polysaccharides from Flammulina velutipes on carbon tetrachloride-induced acute liver injury rats using GC-MS. Int J Biol Macromol 110: 285-293, 2018.

22. Zhang F, Mao Y, Qiao H, Jiang H, Zhao H, Chen X, Tong L and Sun X: Protective effects of taurine against endotoxin-induced acute liver injury after hepatic ischemia reperfusion. Amino Acids 38: 237-245, 2010.

23. Mi H, Muruganujan A, Ebert D, Huang $X$ and Thomas PD: PANTHER version 14: More genomes, a new PANTHER GO-slim and improvements in enrichment analysis tools. Nucleic Acids Res 47 (D1): D419-D426, 2019.

24. Huang da W, Sherman BT and Lempicki RA: Systematic and integrative analysis of large gene lists using DAVID bioinformatics resources. Nat Protoc 4: 44-57, 2009.

25. Fiehn O: Metabolomics by gas chromatography-mass spectrometry: Combined targeted and untargeted profiling. Curr Protoc Mol Biol 114: 30.4.1-30.4.32, 2016.

26. Tavares G, Venturini G,Padilha K, Zatz R, Pereira AC, Thadhani RI, Rhee EP and Titan SMO: 1,5-Anhydroglucitol predicts CKD progression in macroalbuminuric diabetic kidney disease: Results from non-targeted metabolomics. Metabolomics 14: 39, 2018.

27. Chong J, Soufan O, Li C, Caraus I, Li S, Bourque G, Wishart DS and Xia J: MetaboAnalyst 4.0: Towards more transparent and integrative metabolomics analysis. Nucleic Acids Res 46 (W1): W486-W494, 2018.

28. Su G, Morris JH, Demchak B and Bader GD: Biological network exploration with Cytoscape 3. Curr Protoc Bioinformatics 47: 8.13.1-24, 2014

29. Basu S, Duren W, Evans CR, Burant CF, Michailidis G and Karnovsky A: Sparse network modeling and metscape-based visualization methods for the analysis of large-scale metabolomics data. Bioinformatics 33: 1545-1553, 2017.
30. Shou Q, Jin L, Lang J, Shan Q, Ni Z, Cheng C, Li Q, Fu H and Cao G: Integration of metabolomics and transcriptomics reveals the therapeutic mechanism underlying paeoniflorin for the treatment of allergic asthma. Front Pharmacol 9: 1531, 2019.

31. Frenzel C and Teschke R: Herbal hepatotoxicity: Clinical characteristics and listing compilation. Int J Mol Sci 17: pii: E588, 2016.

32. Chiang JYL: Bile acid metabolism and signaling in liver disease and therapy. Liver Res 1: 3-9, 2017.

33. Hylemon PB, Takabe K, Dozmorov M, Nagahashi M and Zhou H: Bile acids as global regulators of hepatic nutrient metabolism. Liver Res 1: 10-16, 2017.

34. de Aguiar Vallim TQ, Tarling EJ and Edwards PA: Pleiotropic roles of bile acids in metabolism. Cell Metab 17: 657-669, 2013.

35. Shimomura Y and Kitaura Y: Physiological and pathological roles of branched-chain amino acids in the regulation of protein and energy metabolism and neurological functions. Pharmacol Res 133: 215-217, 2018.

36. Nelson C: Less methionine means more health. Lab Animal 47 269, 2018.

37. Ding HF and Fisher DE: Mechanisms of p53-mediated apoptosis. Crit Rev Oncog 9: 83-98, 1998.

38. Chen P, Li D, Chen Y, Sun J, Fu K, Guan L, Zhang H, Jiang Y, $\mathrm{Li} X$, Zeng X, et al: p53-mediated regulation of bile acid disposition attenuates cholic acid-induced cholestasis in mice. Br J Pharmacol 174: 4345-4361, 2017.

This work is licensed under a Creative Commons Attribution-NonCommercial-NoDerivatives 4.0 International (CC BY-NC-ND 4.0) License. 